\title{
TOPOLOGICAL CATEGORIES WITH MANY SYMMETRIC MONOIDAL CLOSED STRUCTURES
}

\author{
G.M. Kelly and F. Rossi
}

It would seem from results of Foltz, Lair, and Kelly that symmetric monoidal closed structures, and even monoidal biclosed ones, are quite rare on one-sorted algebraic or essentiallyalgebraic categories. They showed many such categories to admit no such structures at all, and others to admit only one or two; no such category is known to admit an infinite set of such structures.

Among concrete categories, topological ones are in some sense at the other extreme from essentially-algebraic ones; and one is led to ask whether a topological category may admit many such structures. On the category of topological spaces itself, only one such structure - in fact symmetric - is known; although Greve has shown it to admit a proper class of monoidal closed structures. One of our main results is a proof that none of these structures described by Greve, except the classical one, is biclosed.

Our other main result is that, nevertheless, there exist topological categories (of quasi-topological spaces) which admit a proper class of symmetric monoidal closed structures. Even if

Received $23 \mathrm{July} 1984$. The first author gratefully acknowledges the assistance of the Australian Research Grants Scheme, and the second a partial contribution ( $60 \%$ funds) from the MPI, as well as the hospitality of the University of Sydney during November and December 1983.

Copyright Clearance Centre, Inc. Serial-fee code: 0004-9727/85 $\$$ A2. $00+0.00$. 
we insist (like most authors) that topological categories must be wellpowered, we can still exhibit ones with more such structures than any small cardinal.

\section{Introduction}

We recall that a monoidal structure on a category $V$ - given (see [18]) by a "tensor product" functor $\otimes: V \times V \rightarrow V$ and a "unit object" $I$ of $V$, together with natural isomorphisms $a:(X \otimes Y) \otimes Z \cong X \otimes(Y \otimes Z)$, $\tau: I \otimes X \cong X$, and $r: X \otimes I \cong X$, subject to appropriate "coherence axioms" - is said to be closed if each $-\otimes Y: V \rightarrow V$ has a right adjoint $[Y,-]$, and to be biclosed if moreover each $X \otimes-$ has a right adjoint $\{x,-\}$. A symmetric monoidal structure - given by a monoidal one together with a natural isomorphism $c: X \otimes Y \cong Y \otimes X$, related to $a, l$, and $r$ by further coherence axioms - is biclosed if closed, with $\{X,-\}=[X,-]$.

A merely monoidal closed $V$ may well be of interest in itself - [8] for instance makes use of the monoidal closed structure on the category of finitary endofunctors of a locally-finitely-presentable category - but it does not seem to give rise to a fruitful $V$-enriched category theory. The latter is most complete and useful when $V$ is symmetric monoidal closed, and is treated at this level in the recent book [18]; but much of interest survives (see [17] for an example) when $V$ is merely monoidal biclosed.

One is accordingly led to ask whether symmetric monoidal closed structures, or at least monoidal biclosed ones, are common on naturallyoccurring categories $V$, or not. For one-sorted algebraic or essentiallyalgebraic $V$, the results of [10] suggest that such structures are quite rare; seven familiar such $V$ are shown to admit no monoidal biclosed structure, while the categories of abelian groups and abelian monoids admit one each, and the category of categories admits two; all of these four structures being in fact symmetric.

Of course one expects a many-sorted algebraic $V$ to admit more such structures. Indeed the functor-category $V=[K$, Set $]$ is many-sorted algebraic for small $K$, and has by Day [4] as many biclosed monoidal structures as $K$ has "promonoidal structures". The set of these is surely 
small, but by suitable choice of $K$ (see for instance Section 6 below) even the set of symmetric ones can be made larger than any small cardinal.

The same is true if we take instead $V=[K, A b]$. But now $V$, being additive, has a one-object strong generator, and hence a faithful, indeed conservative, right-adjoint functor $U: V \rightarrow$ Set . So the existence of such a $U$ does not in itself impose any restriction, other perhaps than smallness, on the number of symmetric monoidal closed structures. On the other hand, the present $V$, in spite of its one-object generator, cannot be seen as one-sorted algebraic, so long as "algebraic" connotes "finitary operations"; for $U$ is not finitary. No examples seem to be known of one-sorted essentially-algebraic $V$ with more than a finite number of monoidal biclosed structures.

There is a sense in which what some authors (see [16]) call topological categories are at the other extreme from algebraic categories, and it is of interest to ask what the situation is for them. Even merely monoidal closed structures on a topological $V$ necessarily have a very special form: $I$ must be the terminal object, and $X \otimes Y$ must have the same underlying set as the cartesian product $X \times Y$; see Section 3 below.

The paradigmatic topological category is the category Top of topological spaces; Greve [12] has shown that Top admits a large set (that is, a proper class) of monoidal closed structures. Yet no monoidal biclosed structure on Top is known except the one symmetric one in which $[Y, Z]$ is $\operatorname{Top}(Y, Z)$ with the topology of pointwise convergence. $X_{\text {incura }}$ [3] claims to prove that Top admits no other symmetric monoidal closed structure than this one; but his proof seems to us to contain a gap.

Our present results are as follows. We show that none of the monoidal closed structures on Top constructed by Greve, except the classical one, is biclosed. At this point one might begin to suspect that biclosed monoidal structures are as rare on topological categories as on one-sorted essentially algebraic ones, since very few are known on such other topological categories as uniform spaces or compactly-generated spaces. We dispel this suspicion by showing that a certain topological category $q$ Top of "quasi-topological spaces" admits a large set of symmetric monoidal closed structures (as well as a large set of biclosed non-symmetric ones). It is true that $q$ Top is not quite a topological category in the sense of 
[16], lacking the wellpoweredness requirement imposed there. For any small cardinal $\alpha$, however, one can so choose a small full subcategory $A$ of Top that the wellpowered topological $q A$ has more than $\alpha$ symmetric monoidal closed structures.

\section{Isomorphic monoidal structures}

If we are to speak of the number of different structures on $V$, we must know when we count two monoidal structures as "the same". We do so when they are isomorphic, in the sense that we have isomorphisms $\otimes \cong \otimes^{\prime}$ and $I \cong I^{\prime}$ respecting $a, l$, and $r$. No more is required if these structures are closed or biclosed: we automatically have induced isomorphisms $[,] \cong[,]^{\prime}$ and $\{,\} \cong\{,\}^{\prime}$.

Symmetric monoidal structures, however, do not count as the same unless the isomorphisms above also respect $c$. This is truly an extra requirement in general; for the classical monoidal structure on the category of graded abelian groups admits ([9], page 559) two different symmetries $c$ and $c^{\prime}$. This distinction vanishes, however, when $I$ is a generator of $V$, as it always is in our topological examples below. We recall the reasons for this.

LEMMA 2.1. Let $U$ be monoidal closed with $I$ a generator of $V$. Then any $f, f^{\prime}: X \otimes Y \rightarrow Z$ coincide if $f(x \otimes y)=f^{\prime}(x \otimes y): I \otimes I \rightarrow Z$ for alz $x: I \rightarrow X$ and all $y: I \rightarrow Y$.

Proof. Let $f, f^{\prime}$ correspond under the adjunction to $g, g^{\prime}: X \rightarrow[Y, Z]$; so that the composite

$$
I \underset{x}{\longrightarrow} X \underset{g}{\longrightarrow}[Y, Z] \underset{[y, Z]}{\longrightarrow}[I, Z]
$$

is unchanged if $g$ is replaced by $g^{\prime}$. In other words, the composite

$$
V(I, X) \underset{V(I, g)}{\longrightarrow} V(I,[Y, Z]) \underset{V(I,[y, z])}{\longrightarrow} V(I,[I, Z])
$$

is unchanged if $g$ is replaced by $g^{\prime}$. Using the isomorphism

$$
V(I,[Y, Z]) \cong V(I \otimes Y, Z) \cong V(Y, Z)
$$

and the fact that the $V(y, z): V(Y, Z) \rightarrow V(I, Z)$ are jointly monomorphic because $I$ is a generator, we conclude that $V(I, g)=V\left(I, g^{\prime}\right)$, giving $g=g^{\prime}$ since $I$ is a generator; whence $f=f^{\prime}$. 
PROPOSITION 2.2 ([9], Chapter III, Proposition 6.1). Any two symmetries $c, c^{\prime}$ on the monoidal closed $V$ coincide if $I$ is a generator of $V$.

Proof. The naturality of $c$ gives a commutative diagram
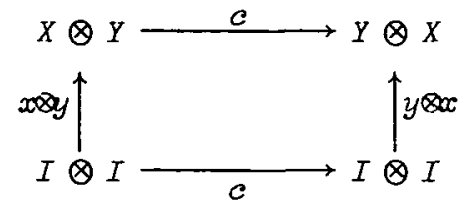

for all maps $x, y$; and since the coherence axioms require $c: I \otimes I \rightarrow I \otimes I$ to be the identity, we have $c(x \otimes y)=c^{\prime}(x \otimes y)$ for all $x, y$; whence $c=c^{\prime}$ by Lemma 2.1.

COROLLARY 2.3. If $V$ has two symmetric monoidal closed stmuctures, any isomorphism of the monoidal structures is an isomorphism of the symmetric monoidal stmuctures, when $I$ is a generator of $V$.

Proof. Transport the symmetry of the $\otimes$-structure along the isomorphism to give a second symmetry on the $\otimes$-structure, and use Proposition 2.2.

\section{Monoidal closed structures on topological categories}

Given a category $V$ with a faithful functor $U: V \rightarrow$ Set, we may always so replace $V$ by an isomorph that each $U: V(X, Y) \rightarrow \operatorname{Set}(U X, U Y)$ is an inclusion of sets; then we may call $(V, U)$ a concrete category, and speak of a function $f: U X \rightarrow U Y$ as a $V$-morphism if it lies in $V(X, Y)$ - as we speak of continuous functions, and so on. An object $X$ of $V$ may then be thought of as a $V$-structure on the set $U X$, counting $X$ and $Y$ with $U X=U Y$ as the same $V$-structure if both $1: X \rightarrow Y$ and $I: Y \rightarrow X$ are $V$-morphisms; on replacing $V$ by an equivalent full subcategory, we may suppose that $X=Y$ in these circumstances.

Such a $(V, U)$ admits initial stmuctures if, given any family (perhaps large) of functions $f_{\alpha}: S \rightarrow U X_{\alpha}$ with $S$ small, there is some $V$-structure $Y$ on $S$ such that each $f_{\alpha}$ is a $V$-morphism $Y \rightarrow X_{\alpha}$ and such that any $g: U Z \rightarrow U Y$ is a $U$-morphism if each $f_{\alpha} g$ is one. Then $(V, U)$ admits final stmuctures as well, the final structure on $S$ for the 
family $h_{\beta}: U W_{B} \rightarrow S$ being the initial structure for the family of all functions $f_{\alpha}: S \rightarrow U X_{\alpha}$ for which each $f_{\alpha} h_{\beta}$ is a $V$-morphism. In particular the empty families give discrete and chaotic structures on any small set $S$, providing left and right adjoints to $U$. Clearly such a $(V, U)$ admits transport of structure along any bijection, while $V$ admits small limits and arbitrary intersections of monomorphisms, as does $V^{o p}$. Both wellpoweredness and cowellpoweredness of $V$ are equivalent to the smallness of the set of structures on any small set $S$.

It is convenient (and clearly possible) to suppose of our category Set of small sets that a function $s: l \rightarrow S$ from the one-element set $I=\{0\}$ is the same thing as an element $s$ of $S$; so that we have not merely an isomorphism but an equality $\operatorname{Set}(1, S)=S$. We call a concrete category $(U, U)$ admitting initial structures topological if the chaotic and discrete structures - and hence all structures - on this set 1 coincide, the corresponding object of $V$ being also called 1 . Then 1 is a terminal object of $V$, and $U=V(1,-): V \rightarrow$ Set . Since $U$ is thus essentially determined by $V$, it is usual to speak of a topological category $U$, without explicit reference to $U$.

The following result, in a version applying to a somewhat wider class

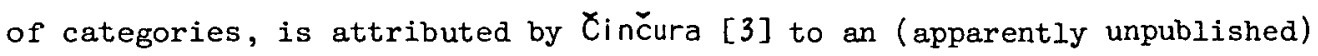
article of Niederle, and has been discussed in greater detail by Pedicchio and Rossi [19].

PROPOSITION 3.1. Any monoidal closed structure on a topological category $V$ is isomorphic to one with the following properties: $I=1$; $U(X \otimes Y)=U X \times U Y ; a, l, r$ are the usual isomorphisms at the level of the underlying sets, and so is $c$ if the structure is symmetric; $U[Y, Z]=V(Y, Z) ;$ and the isomorphism $V(X \otimes Y, Z) \cong V(X,[Y, Z])$ is the restriction of the usual isomorphism

$$
\operatorname{Set}(U X \times U Y, U Z) \cong \operatorname{Set}(U X, \operatorname{Set}(U Y, U Z)) \text {. }
$$

Any isomorphism between two such structures in this canonical form is necessarily the identity.

Proof. In any monoidal $V$, it follows from the naturality of the isomorphisms $l$ and $r$ along with the coherence condition $\tau=r: I \otimes I \rightarrow I$ that the monoid $V(I, I)$ is commutative. Since in a 
topological $V$ we have $U I=V(I, I)$ and $V(I, 1) \cong 1$, we have for each $x \in U I$ the constant map $x !: I \rightarrow I$, with $(x !)(y !)=x !$; so that the commutativity of $U(I, I)$ forces $U I$ to be 0 (empty) or isomorphic to 1 . We can rule out $U I=0$, for then (2.1) gives

$$
V(Y, Z) \cong V(I,[Y, Z]) \subset \operatorname{Set}(0, v[Y, Z]) \cong 1 \text {, }
$$

making $V$ a pre-order, which a topological category is not. So $U I \cong 1$, giving $I \cong 1$; and we may as well take $I=1$.

For any monoidal $V$ we have ([9], Chapter II, Proposition 8.1) a structure of monoidal functor on $V(I,-): V \rightarrow$ Set; so that in our case we have a monoidal functor $\left(U, \tilde{U}, U^{\circ}\right): V \rightarrow$ Set. Here $U^{\circ}: 1 \rightarrow U 1$ is of course the identity; while $\tilde{U}: U X \times U Y \rightarrow U(X \otimes Y)$ sends $(x, y)$ to the composite of $x \otimes y: 1 \otimes 1 \rightarrow X \otimes Y$ with $1 \cong 1 \otimes 1$. We shall show that $\tilde{U}$ is invertible, so that we may as well, by transport of structure, take it to be the identity. Since, as a monoidal functor, $\left(U, \tilde{U}, U^{\circ}\right)$ respects $a, l$, and $r$ (and $c$ too in the symmetric case, as (2.2) shows), the assertions about $a, z, r, c$ will then follow.

Let $W$ be the initial structure on $U X \times U Y$ with respect to the function $\tilde{U}: U X \times U Y \rightarrow U(X \otimes Y)$, so that $\tilde{U}$ is a $V$-morphism $W \rightarrow X \otimes Y$. By Lemma 2.1, this is an epimorphism in $V$; whence, since $U$ has a right adjoint, $\tilde{U}: U X \times U Y \rightarrow U(X \otimes Y)$ is an epimorphism in Set . On the other hand the maps $1 \otimes !: X \otimes Y \rightarrow X \otimes 1 \cong X$ and $! \otimes I: X \otimes Y \rightarrow 1 \otimes Y \cong Y$ give a map $t: X \otimes Y \rightarrow X \times Y$, whose composite with $x \otimes y: I \cong I \otimes I \rightarrow X \otimes Y$ is clearly $(x, y): 1 \rightarrow X \times Y$. In other words $U t . \tilde{U}: U X \times U Y \rightarrow U(X \otimes Y) \rightarrow U(X \times Y) \cong U X \times U Y$ is the identity, so that $\tilde{U}$ is a monomorphism in Set. Hence $\tilde{U}$ is a bijection.

The isomorphism (2.1) here gives $U[Y, Z] \cong V(Y, Z)$, and by transport of structure we may suppose this to be an equality. Because (2.1) is natural, we also have $U[h, k]=V(h, k)$ for maps. If $f: X \otimes Y \rightarrow Z$ corresponds under the adjunction to $g: X \rightarrow[Y, Z]$, then by naturality the composite

$$
1 \otimes 1 \underset{\operatorname{m}}{\longrightarrow} X \otimes Y \underset{f}{\longrightarrow}
$$

corresponds to

$$
1 \underset{x}{\longrightarrow} X \underset{g}{\longrightarrow}[Y, Z] \underset{[y, Z]}{\longrightarrow}[1, Z]
$$


The value of $(U f)(x, y) \in U Z$ is the composite $z$ of $f(x \otimes y)$ with $1 \cong I \otimes I$. Our forcing the isomorphism above to be an equality (in the special case $Y=1$ ) ensures that $(U[y, z] . U g) x$ is this element $z$ of $U[1, Z]=V(1, Z)=U Z$. Since $U[y, z]=V(y, Z)$, this gives $U((U g) x) y=(U f)(x, y)$, as desired.

If $\left(\phi: \otimes \rightarrow \otimes^{\prime}, \psi: 1 \rightarrow 1\right)$ is an isomorphism between two such structures, we necessarily have $\psi=1$; while the naturality of $\phi$ and the compatibility with 2 give commutativity in

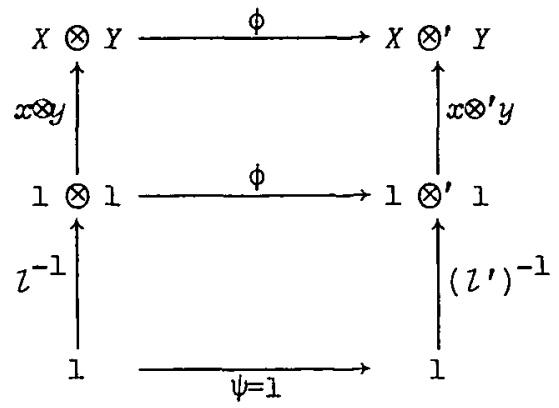

It follows that $U \phi$ is the identity of $U X \times U Y$. Since the same is true of $U\left(\phi^{-1}\right)$, we have $X \otimes Y=X \otimes Y$ with $\phi$ the identity.

\section{Categories of quasi-objects}

Call a locally-small A pretopological if it has a terminal object $I$ which is a generator. We may as well simplify by taking $A$, with the faithful functor $V=A(1,-): A+$ Set, to be concrete, in the sense of the first paragraph of Section 3 , and supposing that $V 1=1$. Every topological category is of course pretopological; while the following well-known construction (see for instance Day [5]) produces from any pretopological A a topological category $q A$ of "quasi-A-objects".

A quasi-A-object $X$ is a small set $S=U X$ with a quasi-A-structure on $U X$. The latter is given by the selection, for each $A \in A$, of a subset $\operatorname{Ad}(A, X)$ of $\operatorname{Set}(V A, U X)$ - the admissible maps - subject to the following two axioms:

$\mathrm{ADI}$

$$
\operatorname{Ad}(1, X)=\operatorname{Set}(1, U X) ;
$$

AD2 if $\phi \in \operatorname{Ad}(B, X)$ and $f \in A(A, B)$ then $\phi . V f \in \operatorname{Ad}(A, X)$. A morphism $g: X \rightarrow Y$ of quasi-objects is a function $g: U X \rightarrow U Y$ such 
that $g \phi \in \operatorname{Ad}(A, Y)$ whenever $\phi \in \operatorname{Ad}(A, X)$. It is immediate that $(q A, U)$ is a topological category.

To each object $B$ of $A$ we associate an object $J B$ of $q A$ with $U J B=V B$ by setting $A d(A, J B)=A(A, B) \subset \operatorname{Set}(V A, V B)$. On taking $A=B$ here we see that $q A(J B, Y)=\operatorname{Ad}(B, Y)$; so that in particular $q A(J B, J C)=A(B, C)$. Thus $J$ identifies $A$ with a full subcategory of $q A$, with $V=U J$.

Note that $q A$ is wellpowered when $A$ is small, while it follows from Example 3.6.6 of [11] that $q$ Top is not wellpowered.

We have the functor $K: q A \rightarrow[A$, Set $]$ given by $K X=q A(J-, X)=A d(-, X)$. Since $K J C=q A(J-, J C)=A(-, C)$, we have $K J=Y$, the Yoneda embedding $A \rightarrow\left[A^{o p}\right.$, Set $]$. It is clear from the definition of morphism in $q A$ that $K$ is fully faithful; so that $A$ is dense in $q A$.

Write $E:\left[A^{o p}\right.$, Set $] \rightarrow$ Set for evaluation at $1 \in A$, noting that $E K=U$. A left adjoint for $E$ is given by the diagonal functor $\Delta$, and a right adjoint by $\Gamma$, where $\Gamma S=\operatorname{Set}\left(V_{-}, S\right)$. The counit of the adjunction $E-\Gamma$ is the identity $E \Gamma=1$; write $n: 1 \rightarrow \Gamma E$ for the unit. Then the $F$-component $\eta_{F}: F \rightarrow \Gamma E F=\operatorname{Set}(V-, F I)$ of $n$ has as its A-component $\left.\left(\eta_{F}\right)_{A}: F A \rightarrow \operatorname{Set}(V A, F]\right)$ the function corresponding to $V A=\mathrm{A}(1, A) \rightarrow \operatorname{Set}(F A, F 1)$.

When $F=K X=\operatorname{Ad}(-, X)$ for $X \in q A$, it is clear that $\eta_{F}$ is a monomorphism, $\left(\eta_{F}\right)_{A}$ being the inclusion $\operatorname{Ad}(A, X) \subset \operatorname{Set}(V A, U X)$. Conversely, if $\eta_{F}$ is a monomorphism, $F$ is isomorphic to $K X$, where $U X=F \perp$ and $\operatorname{Ad}(A, X)$ is the image of $\left(n_{F}\right)_{A}$. It follows at once that a presheaf $F$ is isomorphic to some $K X$ precisely when there is a monomorphism $F+\Gamma S$ for some $S$. The $\Gamma S$ themselves are in effect the quasi-objects with chaotic structure.

If we write

$$
F \underset{\rho_{F}}{\longrightarrow} R F \underset{\sigma_{F}}{\longrightarrow} \Gamma E F
$$


for the epimorphism-monomorphism factorization of $\eta_{F}$, taking $\sigma_{F}$ to be actually an inclusion, then $\rho: 1 \rightarrow R$ is a reflexion of $\left[\mathrm{A}^{\mathrm{op}}\right.$, Set] onto the full subcategory $q A$. We have $U R F=F l$, while $\operatorname{Ad}(A, R F)$ is just the image of $\left(n_{F}\right)_{A}$ in $\operatorname{Set}(V A, F 1)$.

\section{Some monoidal biclosed structures on categories of quasi-objects}

By Proposition 3.1, a monoidal structure $(\otimes, I, \ldots)$ on the pretopological A cannot admit an extension to a biclosed monoidal structure on the topological category $q A$ unless $I \cong I$ and the canonical $\tilde{V}: V A \times V B \rightarrow V(A \otimes B)$, where $V=A(1,-)$, is an isomorphism. These conditions are in fact sufficient: for convenience we suppose the isomorphisms above to be equalities, which they are in our applications. Note that, as in the proof of Proposition 3.1, this forces $a, l, r$ (and $c$ in the symmetric case) to be the usual isomorphisms at the level of underlying sets.

THEOREM 5.1. Let $(\otimes, 1, \ldots)$ be a monoidal structure on the pretopological $A$, for which the canonical $\tilde{V}: V A \times V B \rightarrow V(A \otimes B)$ is an equality. Then the monoidal stmucture extends to one on $q A$ which is biclosed, and which is symmetric if the original one is so.

Proof. Suppose first that $A$ is small. Then by Day [4] we have a "convolution" monoidal biclosed structure $\left(\otimes^{\prime}, I^{\prime}, \ldots\right)$ on $\left[A^{\circ p}\right.$, Set $]$, symmetric if the original one is, given by

$$
\begin{aligned}
I^{\prime} & =\mathrm{A}(-, I), \\
F \otimes^{\prime} G & =\int^{A, B} F A \times G B \times A(-, A \otimes B), \\
{[G, H]^{\prime} } & =\int_{B} \operatorname{Set}(G B, H(-\otimes B)), \\
\{G, H\}^{\prime} & =\int_{B} \operatorname{Set}(G B, H(B \otimes-)) ;
\end{aligned}
$$

the smallness of $A$ ensuring the existence of the right sides of (5.2)-(5.4). When $F$ and $G$ are representables $A(-, C)$ and $A(-, D)$, the Yoneda lemma applied to (5.2) gives $A(-, C) \otimes \otimes^{\prime} A(-, D) \cong A(-, C \otimes D)$. This, with $(5.1)$, shows that $\left(\Theta^{\prime}, I^{\prime}, \ldots\right)$ is an extension of 
$(\otimes, 1, \ldots)$ - at least when we verify that these isomorphisms respect $a, \tau, r$ and (in the symmetric case) $c$, which follows trivially from the definition of these for $\otimes^{\prime}$.

When $G$ in (5.3) is the representable $A(-, A)$, the Yoneda lemma gives $[A(-, A), H]^{\prime} \cong H(A \otimes-)$. If $H=K Z=A d(-, Z)$ for $Z$ in $q A$, we have

$$
\begin{aligned}
{[A(-, A), K Z]^{\prime} \cong \mathrm{Ad}(A \otimes-, Z) \subset \operatorname{Set}(V(A \otimes-), U Z) } & =\operatorname{Set}(V A \times V-, U Z) \\
& \cong \operatorname{Set}(V-, \operatorname{Set}(V A, U Z))=\Gamma(\operatorname{Set}(V A, U Z)) ;
\end{aligned}
$$

so that $[A(-, A), H]^{\prime}$ is isomorphic by section 4 to an object of $q A$. Similarly for $\{A(-, A), H\}^{\prime}$.

It now follows from Cay [5] (see [6] for the non-symmetric case) that we have a monoidal biclosed structure $\left(\otimes^{\prime \prime}, I^{\prime \prime}, \ldots\right)$ - again symmetric if the original one is - on the reflective $q A \subset\left[A^{o p}\right.$, Set], where $X \otimes^{\prime \prime} Y=R\left(K X \otimes^{\prime} K Y\right), I^{\prime \prime}=R I^{\prime}=1,[X, Y]^{\prime \prime}=[K X, K Y]^{\prime}$ which in fact lies in $q^{A}$, and $\{X, Y\}^{\prime \prime}=\{K X, K Y\}^{\prime}$. This is still an extension of $(\otimes, 1, \ldots)$ since, when $X, Y=A, B \in A$, we have

$$
R(K X \otimes K Y)=R(A(-, A) \otimes A(-, B)) \cong R(A(-, A \otimes B)) \cong A \otimes B .
$$

The above completes the proof for small A.. When $A$ is large, we can imitate the above with Set replaced by a category SET of sets in a higher universe with respect to which $A$ is small, getting an extension of the monoidal structure on $A$ to a biclosed monoidal one on the category $Q A$ of quasi-objects with underlying set in SET. We regard Set as a full subcategory of SET, so that $q A$ is the full subcategory of $Q A$ given by the quasi-objects whose underlying set is small.

We complete the proof by showing that $q A$ is closed in $Q A$ for the monoidal biclosed structure. This follows from Proposition 3.1 , since $U\left(X \otimes^{\prime \prime} Y\right)=U X \times U Y, U[Y, Z]^{\prime \prime}=Q A(Y, Z) \subset \operatorname{Set}(U Y, U Z)$, and $U\{Y, Z\}^{\prime \prime} \subset \operatorname{Set}(U Y, U Z) ;$ and all of these are small if $U X, U Y, U Z$ are small.

\section{Some monoidal closed and symetric monoidal structures on Top}

We intend to apply Theorem 5.1 in the case where $A$ is Top, or a full subcategory of Top; accordingly we use $V$ : Top $\rightarrow$ Set for the forgetful functor. We construct monoidal structures on Top using 
techniques of Brown [2], as modified (apparently independently) by Booth and Tillotson [1] and Greve [12] so as to apply to non-hausdorff spaces.

Let $K$ and $L$ be small (to within isomorphism) sets of compact hausdorff spaces, each closed under finite products and hence containing the space 1 . For $A, B \in$ Top consider the family of all continuous $f: K_{f} \rightarrow A$ with domain in $K$ and the family of all continuous $g: L_{g} \rightarrow B$ with domain in $L$. Write $A \otimes B$ for the topological space with underlying set $V A \times V B=V(A \times B)$, but with the final topology with respect to the family given by all $f \times B: K_{f} \times B \rightarrow A \times B$ and all

$A \times g: A \times L_{g} \rightarrow A \times B$. Clearly the map $i: A \otimes B \rightarrow A \times B$, given by the identity on the underlying set, is continuous. It is immediate that $\otimes$ is a functor and $i: \otimes \rightarrow \times$ a natural transformation.

LEMMA 6.1. For $K$ in $K$ the identity map $i: K \otimes B \rightarrow K \times B$ is invertible, and in particular $1 \otimes B=1 \times B \cong B$. Simizarly $A \otimes L=A \times L$ for $L$ in $L$, and $A \otimes 1=A \times 1 \cong A$.

Proof. The map $i^{-1}=I_{K} \times B: K \times B \rightarrow K \otimes B$ is continuous by the definition of $K \otimes B$.

PROPOSITION 6.2. The identity $a:(A \otimes B) \otimes C \rightarrow A \otimes(B \otimes C)$ and $i$ ts inverse are continuous, so that $(\otimes, 1, \ldots)$ is a monoidal stmucture on Top satisfying the hypotheses of Theorem 5.1. This structure is symmetric if $L=K$.

Proof. To say that $A \otimes B$ has the final topology with respect to the maps $f \times B$ and $A \times g$ above is equally to say that these maps exhibit $A \otimes B$ as a quotient space of $\Sigma_{f}\left(K_{f} \times B\right)+\Sigma_{g}\left(A \times L_{g}\right)$. Since product with a compact hausdorff space preserves quotient maps, it follows that $(A \otimes B) \times L^{\prime}$ has, for $L^{\prime} \in \mathrm{L}$, the final topology with respect to the maps $(f \times B) \times L^{\prime}$ and $(A \times g) \times L^{\prime}$. Because $(A \otimes B) \otimes C$ has the final topology with respect to the maps $h \times C: K_{h}^{\prime} \times C \rightarrow(A \otimes B) \times C$ and $(A \otimes B) \times k:(A \otimes B) \times L_{k}^{\prime} \rightarrow(A \otimes B) \otimes C$ where $K_{h}^{\prime} \in K$ and $L_{k}^{\prime} \in \mathrm{L}$, it equally has the final topology with respect to the maps $h \times C: K_{h}^{\prime} \times C \rightarrow A \times B \times C$, the maps $f \times B \times k: K_{f} \times B \times L_{k}^{\prime} \rightarrow A \times B \times C$, and the maps $A \times g \times k: A \times L_{g} \times L_{k}^{\prime} \rightarrow A \times B \times C$. To show that $a$ is 
continuous is to show that all of these maps are continuous when their codomain is taken to be $A \otimes(B \otimes C)$.

Let us dismiss the latter ones first. Since $K_{f} \times B \times L_{k}^{\prime}=K_{f} \otimes\left(B \otimes L_{k}^{\prime}\right)$ by Lemma 6.1, $f \times B \times k$ is the continuous map $f \otimes(B \otimes k): K_{f} \otimes\left(B \otimes L_{k}^{\prime}\right) \rightarrow A \otimes(B \otimes C) ;$ and since $A \times L_{g} \times L_{k}^{\prime}=A \otimes\left(L_{g} \otimes L_{k}^{\prime}\right)$ by Lemma 6.1 and the closedness of $L$ under finite products, $A \times g \times k$ is the continuous map $A \otimes(g \otimes k): A \otimes\left(L_{g} \otimes L_{k}^{\prime}\right) \rightarrow A \otimes(B \otimes C)$.

We turn to $h \times C$ where $h: K_{h}^{\prime} \rightarrow A \otimes B$. Let ih: $K_{h}^{\prime} \rightarrow A \times B$ have components $u: K_{h}^{\prime} \rightarrow A$ and $v: K_{h}^{\prime} \rightarrow B$, and let $d: K_{h}^{\prime} \rightarrow K_{h}^{\prime} \times K_{h}^{\prime}$ be the diagonal. Since $d \times C: K_{h}^{\prime} \times C \rightarrow K_{h}^{\prime} \times K_{h}^{\prime} \times C$ is continuous, it suffices to prove the continuity of $u \times v \times C: K_{h}^{\prime} \times K_{h}^{\prime} \times C+A \otimes(B \otimes C)$. By Lemma 6.1, however, this is the continuous $u \otimes(v \otimes C): K_{h}^{\prime} \otimes\left(K_{h}^{\prime} \otimes C\right) \rightarrow A \otimes(B \otimes C)$.

Thus $a$ is continuous; and similarly $a^{-1}$ is continuous. It is trivial that $c: A \times B \rightarrow B \times A$ is continuous as a map $A \otimes B \rightarrow B \otimes A$ when $L=K$.

We need below an extension of Lemma 6.1. Let us say that a space $A$ is Zocally-K if every point of $A$ has a neighbourhood that lies in $K$. The following is part of [1], Proposition 3.3.

LEMMA 6.3. The map $i: A \otimes B \rightarrow A \times B$ is invertible if $A$ is Zocalzy-K.

Proof. For each $a \in A$ we have $a \in W_{a} \subset K_{a} \subset A$, where $W_{a}$ is open in $A$ and $K_{a} \in K$ is a subspace of $A$. By Lemma 6.1, the inclusion $W_{a} \times B \rightarrow K_{a} \times B=K_{a} \otimes B \rightarrow A \otimes B$ is continuous. Thus if $U$ is open in $A \otimes B$, we have $U \cap\left(W_{a} \times B\right)$ open in $W_{a} \times B$, and hence open in $A \times B$. So $U=\underset{a \in A}{U}\left(U \cap\left(W_{a} \times B\right)\right)$ is open in $A \times B$.

For a cardinal $\alpha$ that is either infinite or 1 , write $c_{\alpha}$ for the 
set of compact hausdorff spaces of cardinality less than or equal to $\alpha$; it is clearly closed under finite products. If $\beta$ and $\alpha$ are two such cardinals, write $\Theta_{B \alpha}$ for the monoidal structure on Top given as above by taking $K=\mathcal{C}_{B}$ and $L=\mathcal{C}_{\alpha}$; it is symmetric by Proposition 6.2 if $\beta=\alpha$.

REMARK 6.4. $A \otimes_{11} B$ is $A \times B$ with the "topology of separate continuity"; this symmetric monoidal structure on Top is closed, $[B, C]$ being $\operatorname{Top}(B, C)$ with the topology of pointwise convergence. As we said in Section 1, no other monoidal biclosed structure on Top is known. Both Booth and Tillotson [1] and Greve [12] observe that each monoidal structure $\otimes_{1 \alpha}$ is closed, $[B, C]$ being $\operatorname{Top}(B, C)$ with the " $C_{\alpha}$-open topology". Greve goes further, in showing that the $Q_{1 \alpha}$ for different $\alpha$ are distinct; he has similar results, at least under the hypothesis that there are no measurable cardinals, for other naturally-occurring topological categories ([13], [14], [15]). We now show that the $\otimes_{\alpha \alpha}$ for different regular $\alpha$ are distinct; observe that the identity $A \otimes_{\beta \beta} B \rightarrow A \otimes_{\alpha \alpha} B$ is continuous for $\beta \leq \alpha$.

PROPOSITION 6.5. For $\beta<\alpha$ and $\alpha$ a regular cardinal, let $A$ be the compact hausdorff space given by the ordinals less than or equal to $\alpha$ with the order-topology. Then the identity $A \otimes_{\beta \beta} A \rightarrow A \otimes_{\alpha \alpha} A$ is not invertible.

Proof. Lemma 6.1 gives $A \Theta_{\alpha \alpha} A=A \times A$, since $A \in C_{\alpha}$. The set $B \subset A \times A$ given by the $(\gamma, \gamma)$ with $\gamma<\alpha$ is not closed in $A \times A$, for $(\alpha, \alpha)$ lies in its closure; but we show $B$ to be closed in $A \Theta_{\beta B} A$.

By symmetry, it suffices to show that $(f \times A)^{-1} B$ is closed in $K \times A$ for each $f: K \rightarrow A$ with $K \in \mathcal{C}_{B}$. Since the subspace $f(K)$ of $A$ is still in $C_{\beta}$, we may as well suppose that $K \subset A$ with $f$ the inclusion. If $\alpha \in K$, the regularity of $\alpha$ shows that $K-\{\alpha\} \subset[0, \gamma]$ for some $\gamma<\alpha$; so that $K=L \cup\{\alpha\}$ for some $L \subset A$ with $L \in \mathcal{C}_{\beta}$. If $\alpha k K$, set $L=K$. Then $(f \times A)^{-1} B=(K \times A) \cap B$ is the diagonal $\Delta$ of $L \times L$; which is closed in $L \times L$ and hence in $K \times A$. 
THEOREM 6.6. There is a Zarge set of symmetric monoidal closed structures on $q$ Top, as well as a large set of non-symmetric monoidal biclosed ones. For any regular cardinal $\alpha$, there is a small full subcategory A of Top such that the wellpowered topological $q \mathrm{~A}$ has at least $\alpha$ symmetric monoidal closed structures.

Proof. The symmetric monoidal closed structures $\otimes_{\alpha \alpha}^{\prime \prime}$ on $q$ Top given by Theorem 5.1 are distinct for different regular $\alpha$, by Proposition 3.1 and Proposition 6.5. Similarly the $\otimes_{I_{\alpha}}^{\prime \prime}$ are distinct by Remark 6.4; that these are indeed non-symmetric for $\alpha>1$ follows from Theorem 7.1 below. For the last statement, let $A$ be the set of topological spaces of cardinal less than or equal to $\alpha$; then $A$ is closed under $\otimes_{\beta \beta}$ for all $\beta$, while by Proposition 6.5 the $\otimes_{\beta \beta}$ for regular $\beta \leq \alpha$ are distinct and there are $\alpha$ such regular cardinals.

\section{Greve's monoidal closed structures on Top are not biclosed}

THEOREM 7.1. None of the monoidal stmuctures $\otimes_{1 \alpha}$ on Top for infinite $\alpha$ is biclosed.

Proof. It suffices to exhibit a topological space $C$ such that $C \otimes_{1 \alpha}$ - does not preserve topological quotient maps; for then it cannot have a right adjoint. Write $\omega$ for the first infinite cardinal. We construct below a quotient map $f: K \rightarrow L$ where $L$ is in $\mathrm{C}_{\omega}$ and $K$ is locally-C $C_{\omega}$, such that not every $C \times f: C \times K \rightarrow C \times L$ is a quotient map. Since $K$ and $L$ are a fortior locally- $C_{\alpha}$, Lemma 6.1 gives $C \times K=C \otimes_{1 \alpha} K$ and $C \times L=C \otimes_{1 \alpha} L$, which completes the proof.

LEMMA 7.2. Let $j: A \rightarrow L$ be a monomorphism (that is, a continuous injection) in Top, and let $L_{j}$ be the set $L$ with a new and finer topology: namely that generated by the sets $G$ which are open in $L$ and the sets $j(H)$ where $H$ is open in $A$. Then this generating set is actually a basis for the topology it generates, and the function $j$ is a continuous map $j^{\prime}: A \rightarrow L_{j}$.

Proof. Since $L$ itself is a generator, and intersections $G \cap G^{\prime}$ 
and $j(H) \cap j\left(H^{\prime}\right)=j\left(H \cap H^{\prime}\right)$ of generators are again generators, we have a basis if $G \cap j(H)$ is a union of generators. But if $y \in G \cap j(H)$ then $y \in j\left(j^{-1}(G) \cap H\right)$, and $j^{-1}(G) \cap H$ is open in $A$. As for the continuity of $j^{\prime}, j^{-1}(G)=j^{-1}(G)$ is open, and $j^{-1} j(H)=H$ is open.

LEMMA 7.3. If $A$ and $L$ in Lemma 7.2 are compact hausdorff, $L_{j}$ is locally-compact hausdorff.

Proof. $L_{j}$ is hausdorff since its topology is finer than that of $L$. Let $y \in L_{j}$. If $y \in j(A)$, it has the compact neighbourhood $j(A)$; for $j(A)$ is open in $L_{j}$, and being $j^{\prime}(A)$ is also compact. If

$y \in L-j(A)$, there is a compact neighbourhood $B$ of $y$ in $L$ not meeting $j(A)$; and $B$ is a fortiom a neighbourhood of $y$ in $L_{j}$. Moreover $B$ is still compact as a subset of $L_{j}$, since a covering of it by basic opens must involve a covering by opens of $L$.

We now take for $A$ the compact subspace of the reals given by 0 and the $1 / n$ for integral $n \geq 1$, and for $L$ the compact subspace of the reals given by 0 , the $1 / n$ for integral $n \geq 1$, and the $1 / n+1 / m$ for integral $n, m \geq 1$. Let $J$ be the set of order-preserving continuous injections $j: A \rightarrow L$ with $j(0)=0$; such a $j$ is in effect a strictly-decreasing sequence in $L-\{0\}$ converging to 0 . We write $K$ for the coproduct $\sum_{j \in J} L_{j}$ in Top, and $f: K \rightarrow L$ for the map whose $j$-component is the identity $L_{j} \rightarrow L$. Obviously $L \in \mathcal{C}_{\omega}$; and $K$ is locally-C $C_{\omega}$ since every element $x$ of $K$ has some $L_{j}$ as a countable neighbourhood, which contains by Lemma 7.3 a countable compact hausdorff neighbourhood of $x$.

LEMMA 7.4. $f: K \rightarrow L$ is a quotient map.

Proof. We have to show that $W \subset L$ is open in $L$ if it is open in each $L_{j}$ with $j \in J$. Let $y \in W$ with $y \neq 0$. Then there is some $j \in J$ with $y \neq j(A)$; so a basic open in $L_{j}$ containing $y$ and contained in $W$ must be of the form $G$ for some open $G$ in $L$, whence $y$ is in the interior of $W$ in $L$. It remains to show that, if $0 \in W$, 
then 0 lies in the interior of $W$ in $L$. Suppose not; then there is some $j \in J$ with $j(A) \cap W=\{0\}$ - which is contradictory since $W$ is open in $L_{j}$ but contains neither a $j(H)$ with $H$ open in $A$ nor a $G$ open in $L$ with $0 \in G$.

LEMMA 7.5. There is a topological space $C$ such that $C \times f: C \times K \rightarrow C \times L$ is not a quatient map.

Proof. The sets $j(A) \subset L_{j}$ for $j \in J$ form an open covering in $K$ of $f^{-1}(0)$. For any finite $\left\{j_{1}, \ldots, j_{p}\right\}$ in $J$, the set $D=j_{1}(A) \cup \ldots \cup j_{p}(A)$ is not a neighbourhood of 0 in $L$; since $D$ contains for each $n$ only a finite number of elements exceeding $1 / n$, while any neighbourhood of 0 in $L$ contains, for some $n$, an infinite number of elements exceeding $1 / n$. The result now follows from Theorem 3 and Proposition 4 of [7].

\section{References}

[1] P. Booth and J. Tillotson, "Monoidal closed, cartesian closed and convenient categories of topological spaces", Pacific J. Math. $88(1980), 35-53$.

[2] R. Brown, "Ten topologies for $X \times Y$ ", Quart. J. Math. Oxford. Ser. (2) $14(1963), 303-319$.

[3] Juraj Cinčura, "Tensor products in the category of topological spaces", Comment. Math. Univ. Carolin. 20 (1979), 431-446.

[4] Brian Day, "On closed categories of functors", Reports of the Michest Category Seminar IV, I-38 (Lecture Notes in Mathematics, 137. Springer-Verlag, Berlin, Heidelberg, New York, 1970).

[5] Brian Day, "A reflection theorem for closed categories", J. Pure App 2. Algebra 2 (1972), 1-11.

[6] Brian Day, "On closed categories of functors II", Category Seminar, 20-54 (Proc. Sydney Category Theory Seminar, 1972/1973. Lecture Notes in Mathematics, 420. Springer-Verlag, Berlin, Heidelberg, New York, 1974). 
[7] B.J. Day and G.M. Kelly, "On topological quotient maps preserved by pullbacks or products", Proc. Cambridge Philos. Soc. 67 (1960), 553-558.

[8] E.J. Dubuc and G.M. Kelly, "A presentation of topoi as algebraic relative to categories or graphs", J. Algebra 81 (1983), $420-433$.

[9] Samuel Eilenberg and G.M. Kelly, "Closed categories", Proc. Conf. on Categorical AZgebra (La Jolla 1965), 421-562 (Springer-Verlag, Berlin, Heidelberg, New York, 1966).

[10] F. Foltz, C. Lair, and G.M. Kelly, "Algebraic categories with few biclosed monoidal structures or none", J. Pure Appl. Algebra 17 (1980), 171-177.

[11] P.J. Freyd and G.M. Kelly, "Categories of continuous functors I", $J$. Pure App 2. Algebra 2 (1972), 169-191.

[12] Georg Greve, "How many monoidal closed structures are there in TOP?", Arch. Math. (Basel) 34 (1980), 538-539.

[13] Georg Greve, "An extension theorem for monoidal closed topological categories", Seminarberichte aus dem Fachbereich Mathematik der Fermuniversität 7, 107-119 (Hagen, 1980).

[14] Georg Greve, "Rigid spaces and monoidal closedness", Categorical Aspects of Topology and Analysis; Proceedings 1981, 105-111 (Lecture Notes in Mathematics, 915. Springer-Verlag, Berlin, Heidelberg, New York, 1982).

[15] Georg Greve, "General construction of monoidal closed structures on topological, uniform and nearness spaces", Category Theory; Applications to Algebra, Logic and Topology; Proceedings, Gummersbach, 1981, 100-114 (Lecture Notes in Mathematics, 962. Springer-Verlag, Berlin, Heidelberg, New York, 1982).

[16] Horst Herrlich, "Categorical topology 1971-1981", General Topology and its Relations to Modern Analysis and Algebra; Proc. 5th Prague Topol. Symposium 1981, 279-383 (Sigma Ser. Pure Math. 3). 
[17] S. Kasangian, G.M. Kelly, and F. Rossi, "Cofibrations and the realization of non-deterministic automata", Cahiers Topologie Géom. Differentielze 24 (1983), 23-46.

[18] G.M. Kelly, Basic concepts of enriched category theory (London Mathematical Society Lecture Notes Series 64. Cambridge University Press, Cambridge, 1982).

[19] M. Cristina Pedicchio and F. Rossi, "Some remarks on categories with constant maps and monoidal closedness", Rend. Istit. Mat. Univ. Trieste (to appear).

Department of Pure Mathematics, University of Sydney,

Sydney,

New South Wales 2006,

Australia;

Istituto di Matematica,

Università degli Studi di Trieste,

34100 Trieste,

Italy. 